Supporting Information of

\title{
Integration of Kinetic Control and Lattice Mismatch to Synthesize Pd@AuCu Core-Shell Planar Tetrapods with Size-Dependent Optical Properties
}

\author{
Min Meng, ${ }^{\dagger, \S}$ Zhicheng Fang, ${ }^{\dagger, \S}$ Chao Zhang, ${ }^{\ddagger}$ Hongyang Su, ${ }^{\S}$ Rong He, ${ }^{\S}$ Renpeng Zhang, ${ }^{\S}$
} Hongliang Li,${ }^{\S}$ Zhi-Yuan $\mathrm{Li},{ }^{\ddagger}$ Xiaojun $\mathrm{Wu},{ }^{\S}$ Chao Ma $,{ }^{*},{ }^{\S}$ and Jie Zeng ${ }^{*}{ }^{\S}$

${ }^{\S}$ Hefei National Laboratory for Physical Sciences at the Microscale, Key Laboratory of Strongly-Coupled Quantum Matter Physics of Chinese Academy of Sciences, Hefei Science Center \& National Synchrotron Radiation Laboratory, Department of Chemical Physics, University of Science and Technology of China, Hefei, Anhui 230026, P. R. China Laboratory of Optical Physics, Institute of Physics, Chinese Academy of Sciences, P. O. Box 603, Beijing 100190, P. R. China

\footnotetext{
*To whom correspondence should be addressed. E-mail: zengj@ustc.edu.cn (JZ), cma@ustc.edu.cn (CM)
}

$\dagger$ These authors contributed equally. 


\section{Experimental Section}

Chemicals and materials. Copper(II) chloride dihydrate $\left(\mathrm{CuCl}_{2} \cdot 2 \mathrm{H}_{2} \mathrm{O}, 99 \%\right)$, copper(II) acetylacetonate $\left(\mathrm{Cu}(\mathrm{acac})_{2}, 98.5 \%\right)$, tetrachloroaurate trihydrate $\left(\mathrm{HAuCl}_{4} \cdot 3 \mathrm{H}_{2} \mathrm{O}, 99 \%\right)$, cetyltrimethylammonium chloride (CTAC, >98\%), palladium(II) chloride ( $\mathrm{PdCl}_{2}$, anhydrous), cetyltrimethylammonium bromide (CTAB, $>98 \%$ ), glucose ( $\alpha$ or $\beta$ form), potassium bromide (KBr, >99\%), 1-dodecanethiol (DDT, 98\%), L-ascorbic acid (AA, >99\%), sodium hydroxide $(\mathrm{NaOH},>96 \%)$, formaldehyde (HCHO, 37\% 40\% aqueous solution), hydrochloric acid ( $\mathrm{HCl}$, $36 \% \sim 38 \%$ aqueous solution), crystal violet (CV, >99\%), and diphenyl ether (DPE, 98\%) were purchased from Sinopharm Chemical Reagent Co. Ltd (Shanghai, China). Hexadecylamine (HDA, 90\%), poly (vinylpyrrolidone) (PVP, $\left.\mathrm{M}_{\mathrm{w}} \approx 55,000\right)$, 1,2-hexadecanediol (HDD, 90\%), 1-adamantanecarboxylic acid (ACA, 99\%), and sodium tetrachloropalladate(II) $\left(\mathrm{Na}_{2} \mathrm{PdCl}_{4}, 98 \%\right.$ ) were obtained from Sigma-Aldrich. All chemical reagents were used as received without further purification. Ultrapure Millipore water $(18.2 \mathrm{M} \Omega \cdot \mathrm{cm})$ was used as the solvent throughout. All the vials were made from borosilicate glass, with a white phenolic moulded screw cap and polyvinyl-faced pulp liner.

Synthesis of 9-nm Pd cubic seeds. The Pd cubic seeds with an average aspect ratio of 1.2 were synthesized according to previous procedures. ${ }^{1}$ Specifically, $8.0 \mathrm{~mL}$ of an aqueous solution containing $105 \mathrm{mg}$ of PVP, $60 \mathrm{mg}$ of $\mathrm{AA}$, and $300 \mathrm{mg}$ of $\mathrm{KBr}$ was added into a vial and preheated to $80{ }^{\circ} \mathrm{C}$ in an oil bath under magnetic stirring for $10 \mathrm{~min}$. Subsequently, $3.0 \mathrm{~mL}$ of an aqueous solution containing $57 \mathrm{mg}$ of $\mathrm{Na}_{2} \mathrm{PdCl}_{4}$ was added into the vial with a pipette. After the vial had been capped, the reaction was allowed to continue at $80{ }^{\circ} \mathrm{C}$ for $3 \mathrm{~h}$. After being washed with water three times by centrifugation, the Pd cubic seeds were stored in $10 \mathrm{~mL}$ of PVP aqueous solution $\left(1 \mathrm{mg} \mathrm{mL}^{-1}\right)$ for future use $\left(\sim 1.9 \mathrm{mg} \mathrm{mL}^{-1}\right.$ in Pd element).

Synthesis of 22-nm Pd cubic seeds. The Pd cubic seeds with an aspect ratio of $\sim 1.0$ were synthesized according to Xu's work. ${ }^{2}$ Firstly, an aqueous solution containing $\mathrm{H}_{2} \mathrm{PdCl}_{4}(10 \mathrm{mM})$ was prepared by dissolving $177.3 \mathrm{mg}$ of $\mathrm{PdCl}_{2}$ in $10 \mathrm{~mL}$ of an aqueous solution containing $\mathrm{HCl}$ $(0.2 \mathrm{M})$ and further diluting to $100 \mathrm{~mL}$ with ultrapure water. Then $0.5 \mathrm{~mL}$ of an aqueous solution containing $\mathrm{H}_{2} \mathrm{PdCl}_{4}(10 \mathrm{mM})$ was added to $10 \mathrm{~mL}$ of an aqueous solution containing CTAB (12.5 mM) under stirring. Afterwards, the solution was heated at $95{ }^{\circ} \mathrm{C}$ for $5 \mathrm{~min}$ before $80 \mu \mathrm{L}$ of a freshly prepared aqueous solution containing AA $(100 \mathrm{mM})$ was added. The reaction was allowed to proceed at $95^{\circ} \mathrm{C}$ for $30 \mathrm{~min}$. After being precipitated by centrifugation at 13,000 rpm 
for $10 \mathrm{~min}$, the Pd cubic seeds were stored in $0.4 \mathrm{~mL}$ of PVP aqueous solution $\left(1 \mathrm{mg} \mathrm{mL}^{-1}\right)$ for future use $\left(\sim 1.22 \mathrm{mg} \mathrm{mL}^{-1}\right.$ in Pd element).

Synthesis of 12-nm $\mathrm{Au}_{3} \mathrm{Cu}$ nanocubes. The $\mathrm{Au}_{3} \mathrm{Cu}$ nanocubes were prepared according to previous procedures. ${ }^{3}$ Specifically, a mixture of $\mathrm{HAuCl}_{4}(85 \mathrm{mg}, 0.248 \mathrm{mmol}), \mathrm{Cu}(\mathrm{acac})_{2}(65 \mathrm{mg}$, $0.25 \mathrm{mmol}$ ), DDT (0.5 mL, $2.08 \mathrm{mmol})$, ACA (270 mg, $3.36 \mathrm{mmol})$, HDD (1600 mg, 6.19 mmol), and HDA (2000 mg, $8.28 \mathrm{mmol}$ ) was added in a three-neck, round-bottom flask with 10 $\mathrm{mL}$ of DPE as the solvent. The reaction mixture was heated at a rate of $8{ }^{\circ} \mathrm{C} \mathrm{min}^{-1}$ and held at $180{ }^{\circ} \mathrm{C}$ for 5 hours. After the final solution was cooled down, the products were separated from organic residues by first centrifugation at 13,000 rpm for $10 \mathrm{~min}$, and then washed with chloroform three times and ethanol once. The products were finally dispersed in ethanol with the aid of sonication for future use $\left(\sim 4.21 \mathrm{mg} \mathrm{mL}^{-1}\right.$ in $\mathrm{Au}$ element and $\sim 0.45 \mathrm{mg} \mathrm{mL}^{-1}$ in $\mathrm{Cu}$ element).

Synthesis of Pd@AuCu core-shell planar tetrapods. In a standard synthesis, $0.3 \mathrm{~mL}$ of an aqueous solution containing $\mathrm{CuCl}_{2} \cdot 2 \mathrm{H}_{2} \mathrm{O}(100 \mathrm{mM}), 0.3 \mathrm{~mL}$ of an aqueous solution containing $\mathrm{HAuCl}_{4} \cdot 3 \mathrm{H}_{2} \mathrm{O}(100 \mathrm{mM}), 90 \mathrm{mg}$ of HDA, $0.2 \mathrm{~mL}$ of an aqueous solution containing CTAC (1 M) and $3.76 \mathrm{~mL}$ of water were added in a $20-\mathrm{mL}$ vial at room temperature. After the vial had been capped, the solution was magnetically stirred at room temperature overnight. Then, $0.3 \mathrm{~mL}$ of 9-nm Pd cubic seeds $\left(1.9 \mathrm{mg} \mathrm{mL}^{-1}\right)$ and $0.14 \mathrm{~mL}$ of an aqueous solution containing glucose $(1 \mathrm{M})$ were added into the vial using a pipette and stirred for $5 \mathrm{~min}$. The capped vial was then transferred into an oil bath and heated at $95{ }^{\circ} \mathrm{C}$ for 30 min under magnetic stirring. As the reaction proceeded, the solution changed its color from kelly green to dark blue. Then the products were centrifugated at 8,000 rpm for $10 \mathrm{~min}$ and washed with water three times and ethanol twice to remove excess precursors, HDA, and glucose. The sizes of $\mathrm{Pd} @ \mathrm{AuCu}$ nanocrystals could be controlled by simply adjusting the amounts of $\mathrm{Pd}$ cubic seeds with the other conditions unchanged. In details, 70-nm, 44-nm, and 33-nm planar tetrapods were synthesized with the addition of $0.1,0.3$, and $0.5 \mathrm{~mL}$ of $\mathrm{Pd}$ seeds, respectively. The three-dimensional $\mathrm{Pd} @ \mathrm{AuCu}$ multipods were synthesized with the addition of $0.3 \mathrm{~mL}$ of $\mathrm{Pd}$ cubic seeds with an aspect ratio of $\sim 1.0$ instead of previous Pd cubic seeds with the other conditions unchanged. The $\mathrm{Au}_{3} \mathrm{Cu} @ \mathrm{AuCu}$ core-shell nanocrystals were synthesized with the addition of $0.3 \mathrm{~mL}$ of $\mathrm{Au}_{3} \mathrm{Cu}$ cubic seeds instead of $\mathrm{Pd}$ cubic seeds with the other conditions unchanged. 
Characterizations. Transmission electron microscopy (TEM) images were taken using a Hitachi $\mathrm{H}-7650$ transmission electron microscope at an acceleration voltage of $100 \mathrm{kV}$. High-angle annular dark-field scanning TEM (HAADF-STEM), and energy dispersive X-ray (EDX) analyses were collected on a JEOL ARM-200F field-emission transmission electron microscope operating at $200 \mathrm{kV}$ accelerating voltage using Mo-based TEM grids. X-ray diffraction (XRD) characterization was performed using a Philips X'Pert Pro X-ray diffractometer with a monochromatized $\mathrm{Cu} \mathrm{K} \alpha$ radiation source and a wavelength of $0.1542 \mathrm{~nm}$. Inductive coupled plasma-atomic emission spectroscopy (ICP-AES, Atomscan Advantage, Thermo Jarrell Ash, USA) was used to determine the concentration of $\mathrm{Pd}, \mathrm{Au}$, and $\mathrm{Cu}$. Extinction spectra were recorded on a U-4100 at room temperature (Hitachi, Japan). Fourier transform infrared (FT-IR)

spectra were measured on a Bruker Vector-22 FT-IR spectrometer from 400 to $4000 \mathrm{~cm}^{-1}$ at room temperature. Raman spectra were obtained on a JYLABRAM-HR spectrometer equipped with an integral microscope.

\section{REFERENCES}

(1) Jin, M.; Liu, H.; Zhang, H.; Xie, Z.; Liu, J.; Xia, Y. Nano Res. 2011, 4, 83-91.

(2) Niu, W.; Li, Z. Y.; Shi, L.; Liu, X.; Li, H.; Han, S.; Chen, J.; Xu, G. Cryst. Growth Des. 2008, $8,4440-4444$.

(3) Liu, Y.; Walker, A. R. Angew. Chem. Int. Ed. 2010, 122, 6933-6937. 

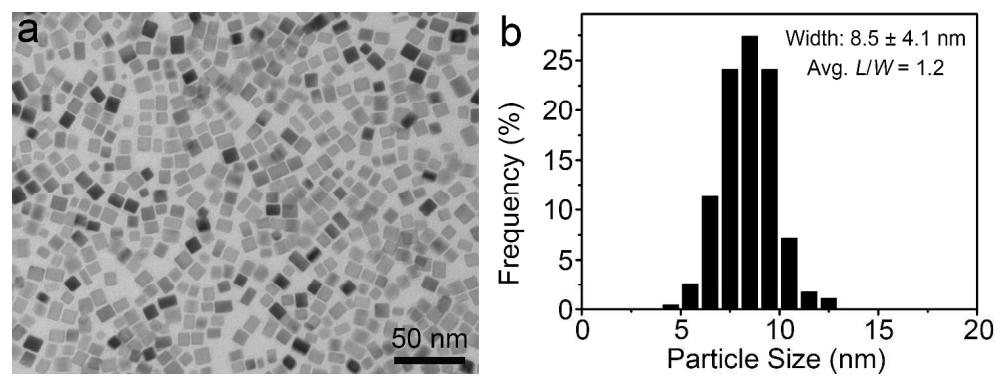

Figure S1. (a) TEM image of 9-nm Pd cubic seeds. (b) The corresponding histogram of size distribution for Pd cubic seeds. For the cubes, both width and average aspect ratio are presented to describe their sizes. The number of particles is over 600 in the statistical measurement. 


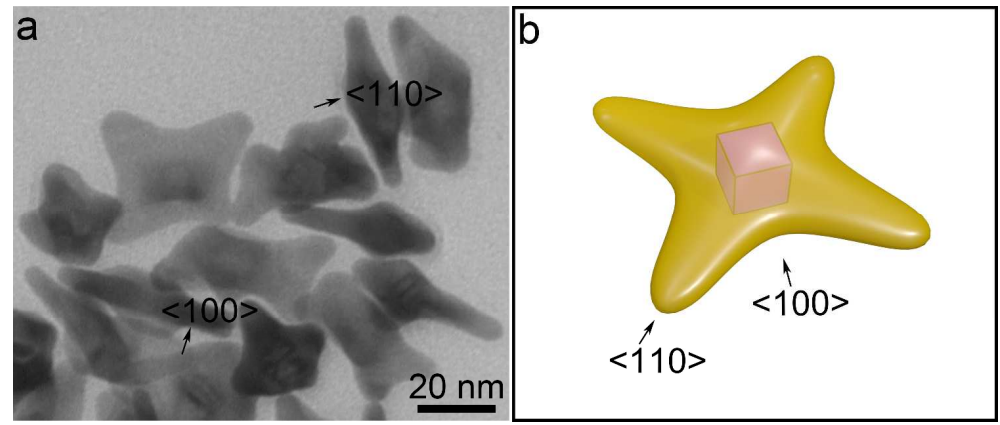

Figure S2. (a) TEM image of $\mathrm{Pd} @ \mathrm{AuCu}$ core-shell planar tetrapods viewed from different axes. (b) Geometric model of an individual $\mathrm{Pd} @ \mathrm{AuCu}$ core-shell planar tetrapod and corresponding directions. 


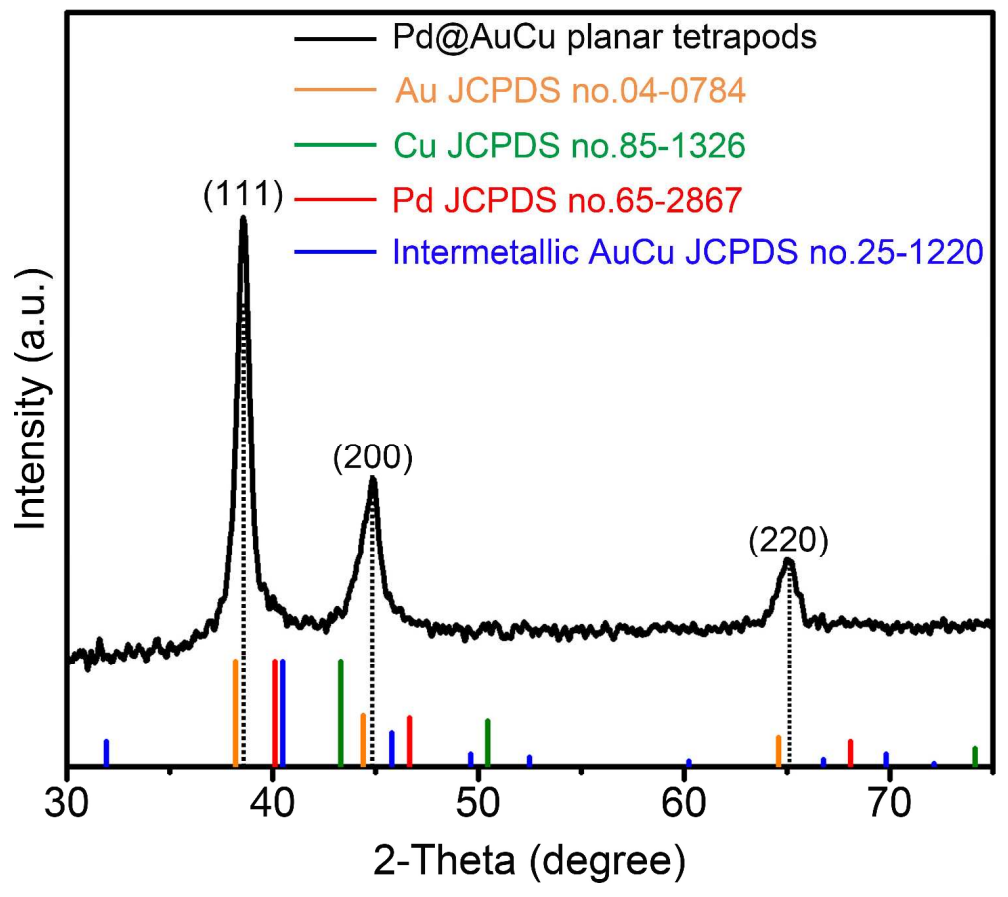

Figure S3. XRD pattern of Pd@AuCu core-shell planar tetrapods prepared through the standard procedure. The standard diffraction peaks of pure $\mathrm{Pd}, \mathrm{Au}, \mathrm{Cu}$, and intermetallic $\mathrm{AuCu}$ are also presented at the bottom for comparison. 


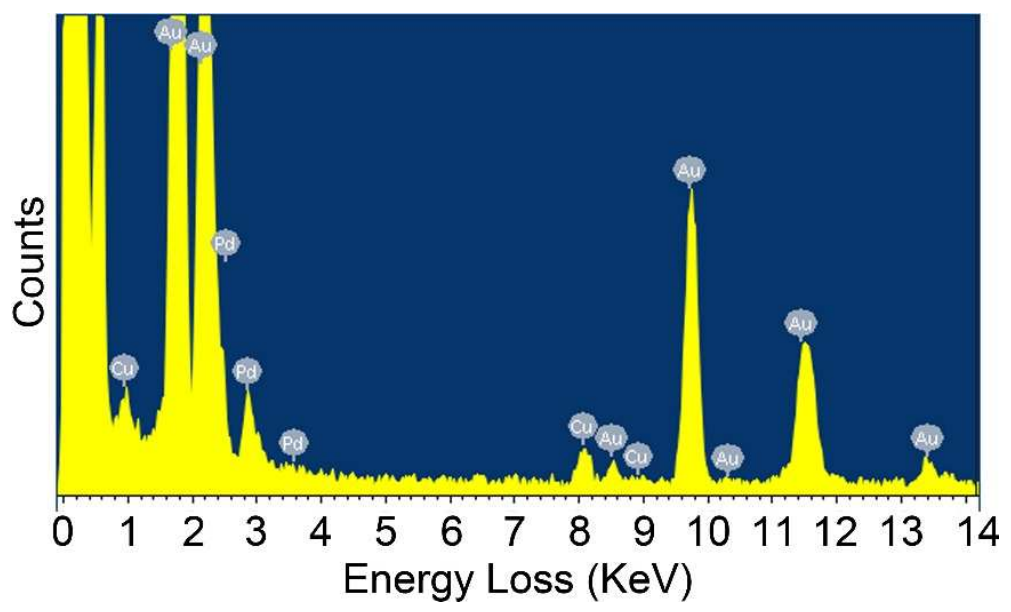

Figure S4. EDX spectrum of an individual Pd@AuCu core-shell planar tetrapod shown in Figure 2a. 


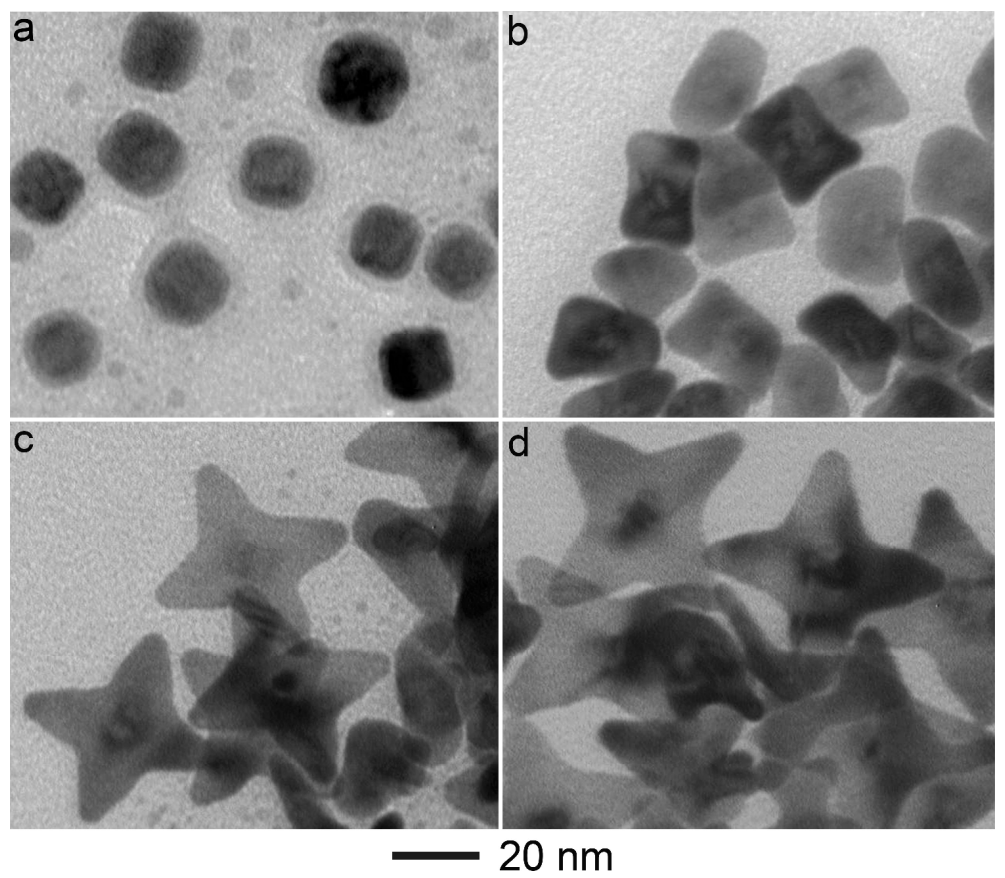

Figure S5. TEM images of $\mathrm{Pd@AuCu}$ core-shell planar tetrapods obtained at different reaction time points in the standard synthetic procedure: (a) $t=2 \mathrm{~min}$, (b) $t=4 \mathrm{~min}$, (c) $t=8 \mathrm{~min}$, and (d) $\mathrm{t}=30 \mathrm{~min}$. 


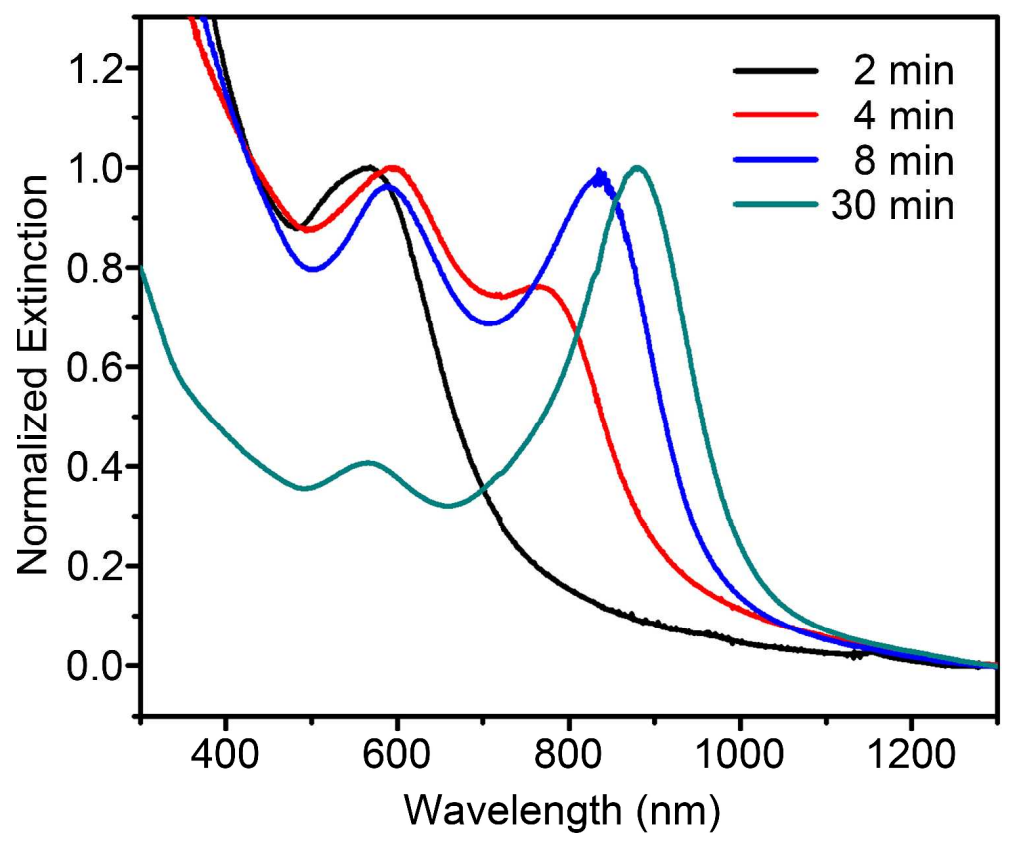

Figure S6. UV-Vis spectra of the reaction solution obtained at different reaction time points in the standard procedure. 

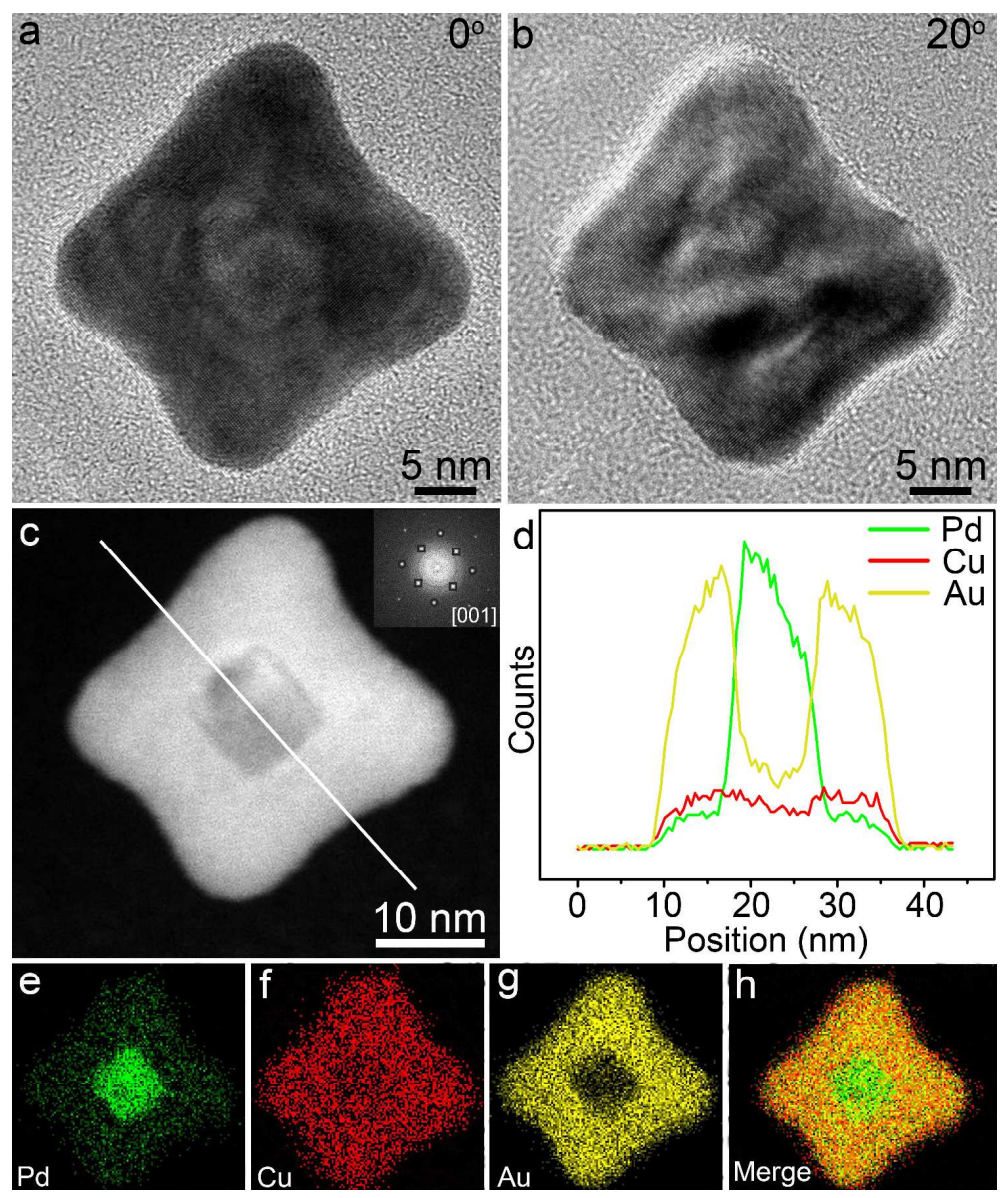

Figure S7. (a, b) TEM images of an individual $\mathrm{Pd} @ \mathrm{AuCu}$ nanocrystal obtained at $\mathrm{t}=4 \mathrm{~min}$ recorded at different tilting angles: (a) $0^{\circ}$ and (b) $20^{\circ}$. (c) HAADF-STEM image of the nanocrystal shown in (a). The inset shows the corresponding SAED pattern with the electron beam directed along the [001] axis. The circles correspond to electron diffractions from the $\{220\}$ planes while the boxes correspond to those from the $\{200\}$ planes. (d) Elemental line-scanning profiles along the direction marked by a white line in (c). (e-h) STEM-EDX elemental mapping of (e) $\mathrm{Pd},(\mathrm{f}) \mathrm{Cu},(\mathrm{g}) \mathrm{Au}$, and (h) merged images of the nanocrystal shown in (c). 


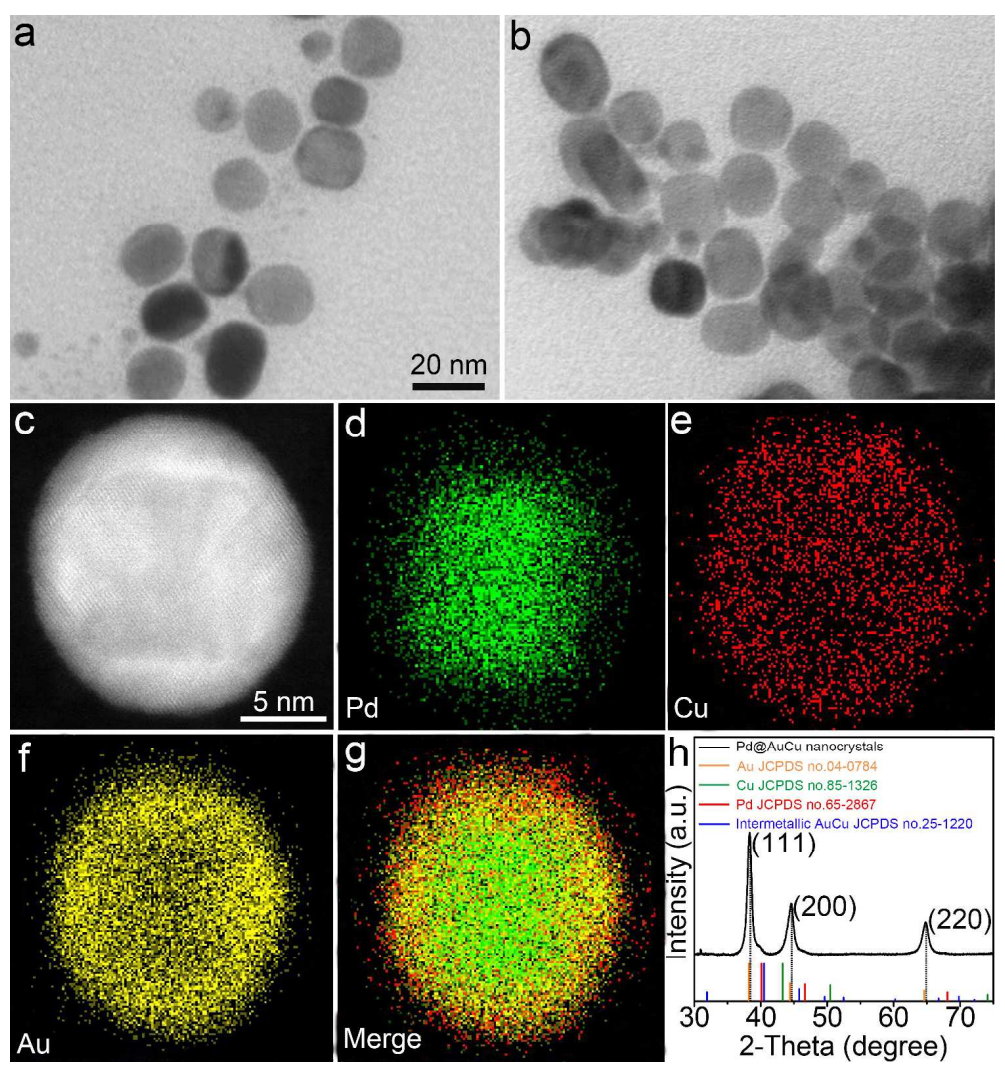

Figure S8. $(\mathrm{a}, \mathrm{b})$ TEM images of the products obtained through the standard procedure except for (a) adding $20 \mu \mathrm{L}$ of $1 \mathrm{M} \mathrm{NaOH}$ aqueous solution and (b) using $10 \mu \mathrm{L}$ of HCHO solution to replace glucose. (c) HAADF-STEM image of an individual spherical Pd@AuCu core-shell nanocrystal in (b). (d-g) STEM-EDX elemental mapping of (d) $\mathrm{Pd}$, (e) $\mathrm{Cu}$, (f) Au, and (g) merged images of the nanocrystal shown in (c). (h) XRD pattern of spherical $\mathrm{Pd} @ \mathrm{AuCu}$ core-shell nanocrystals shown in (b). The standard diffraction peaks of pure $\mathrm{Pd}, \mathrm{Au}, \mathrm{Cu}$, and intermetallic $\mathrm{AuCu}$ are also presented at the bottom for comparison. The scale bar in (a) also applies to (b). 


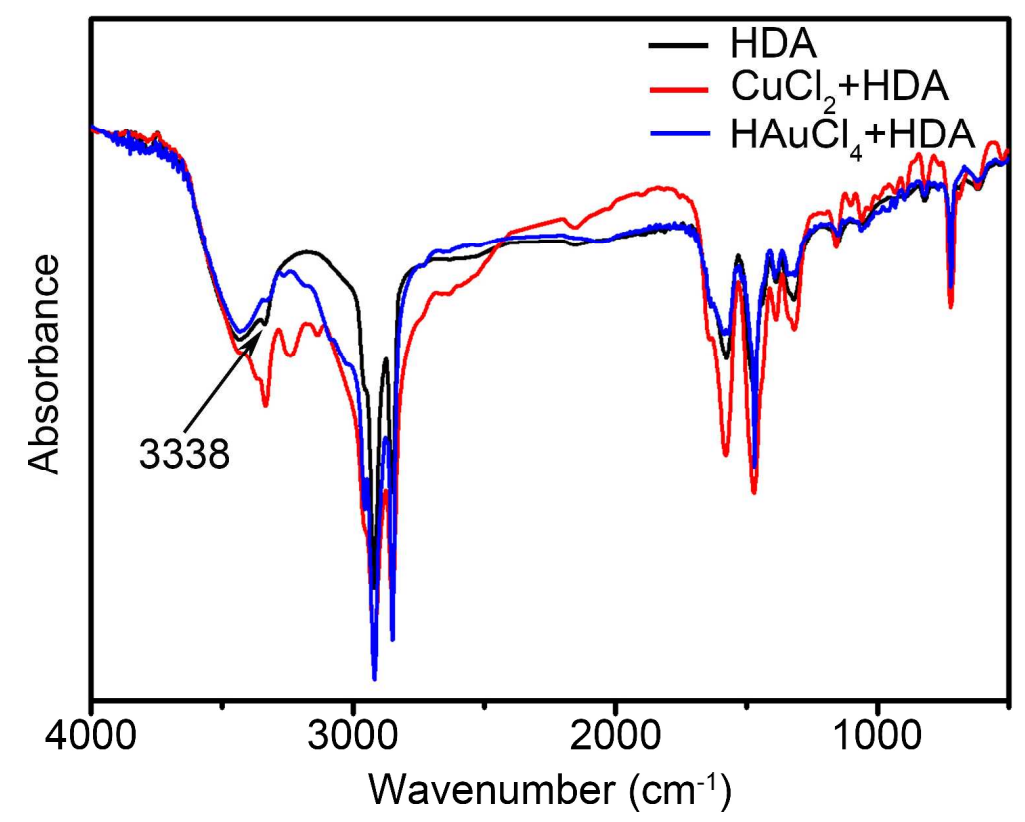

Figure S9. FT-IR characterizations of HDA (black line), $\mathrm{Cu}^{2+}$-HDA complex (red line), and $\mathrm{Au}^{3+}$-HDA complex (blue line). 


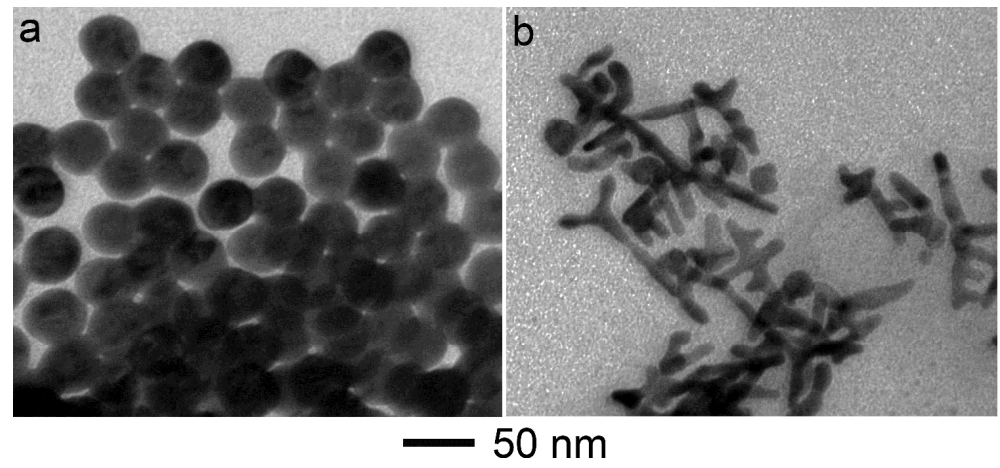

Figure S10. TEM images of the products obtained through the standard procedure except for using different amounts of HDA: (a) $45 \mathrm{mg}$ and (b) $180 \mathrm{mg}$. 


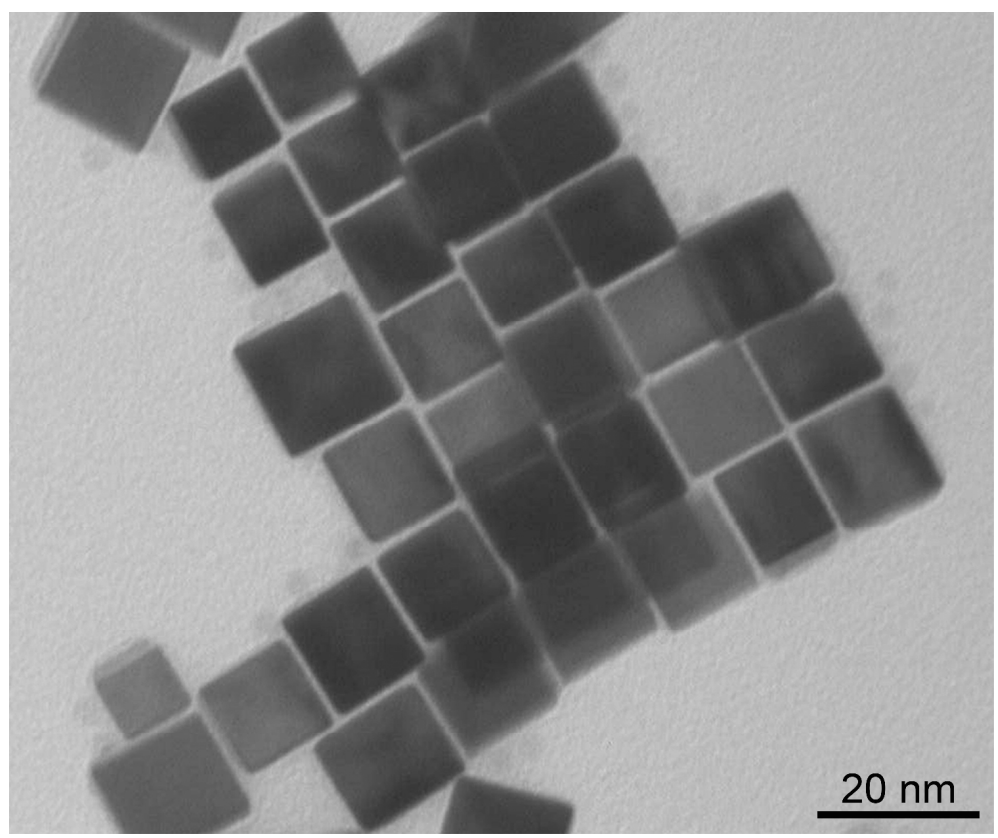

Figure S11. TEM image of $12-\mathrm{nm} \mathrm{Au}_{3} \mathrm{Cu}$ nanocubes. 

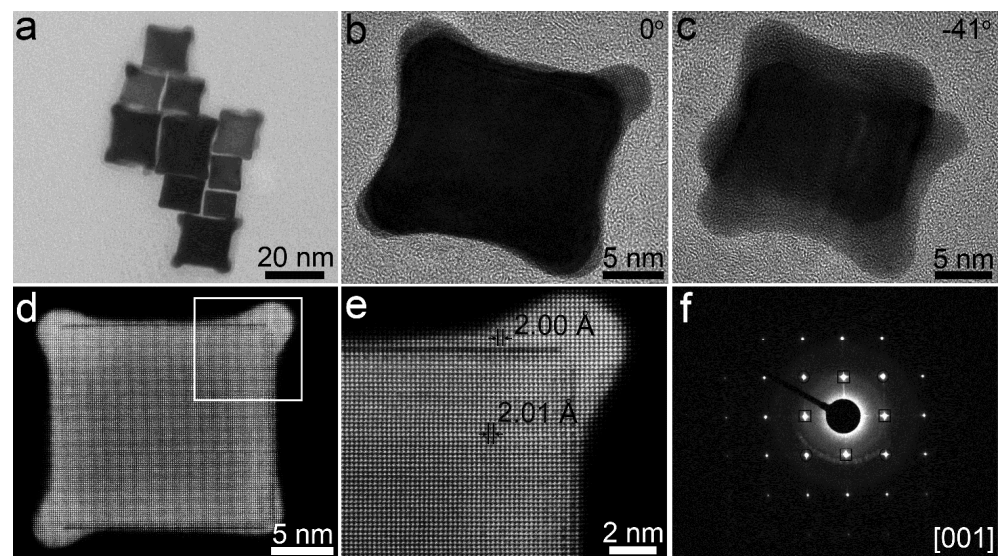

Figure S12. (a) TEM image of $\mathrm{Au}_{3} \mathrm{Cu} @ \mathrm{AuCu}$ nanocrystals prepared through the standard procedure except for using 12-nm $\mathrm{Au}_{3} \mathrm{Cu}$ nanocubes to replace 9-nm Pd cubic seeds. (b, c) TEM images of an individual $\mathrm{Au}_{3} \mathrm{Cu} @ \mathrm{AuCu}$ nanocrystal recorded at different tilting angles: (b) $0^{\circ}$ and (c) $-41^{\circ}$. (d) HAADF-STEM image of an individual $\mathrm{Au}_{3} \mathrm{Cu} @ \mathrm{AuCu}$ nanocrystal. (e) Corresponding HAADF-STEM image of the region marked in (d). (f) SAED pattern of the nanocrystal in (d) with the electron beam directed along the [001] axis. The circles correspond to electron diffractions from the $\{220\}$ planes while the boxes correspond to those from the $\{200\}$ planes. 

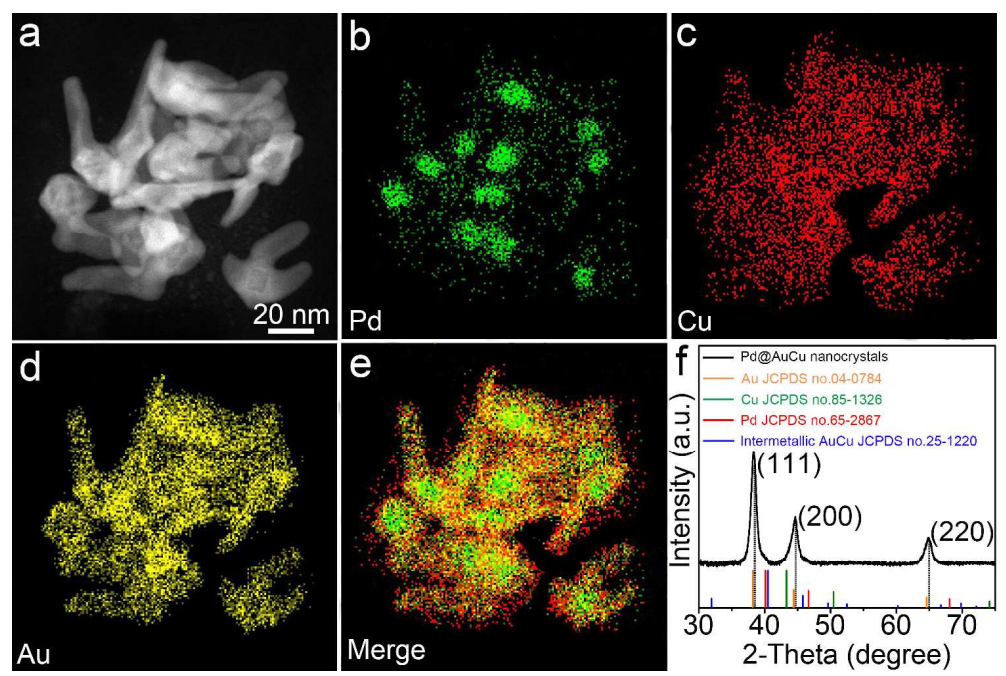

Figure S13. (a) HAADF-STEM image of $\mathrm{Pd} @ \mathrm{AuCu}$ core-shell nanocrystals obtained through the standard procedure without the addition of CTAC. (b-e) STEM-EDX elemental mapping of (b) $\mathrm{Pd},(\mathrm{c}) \mathrm{Cu}$, (d) $\mathrm{Au}$, and (e) merged images of the nanocrystals shown in (a). (f) XRD pattern of the Pd@AuCu core-shell nanocrystals. The standard diffraction peaks of pure $\mathrm{Pd}, \mathrm{Au}, \mathrm{Cu}$, and intermetallic $\mathrm{AuCu}$ are also presented at the bottom for comparison. 

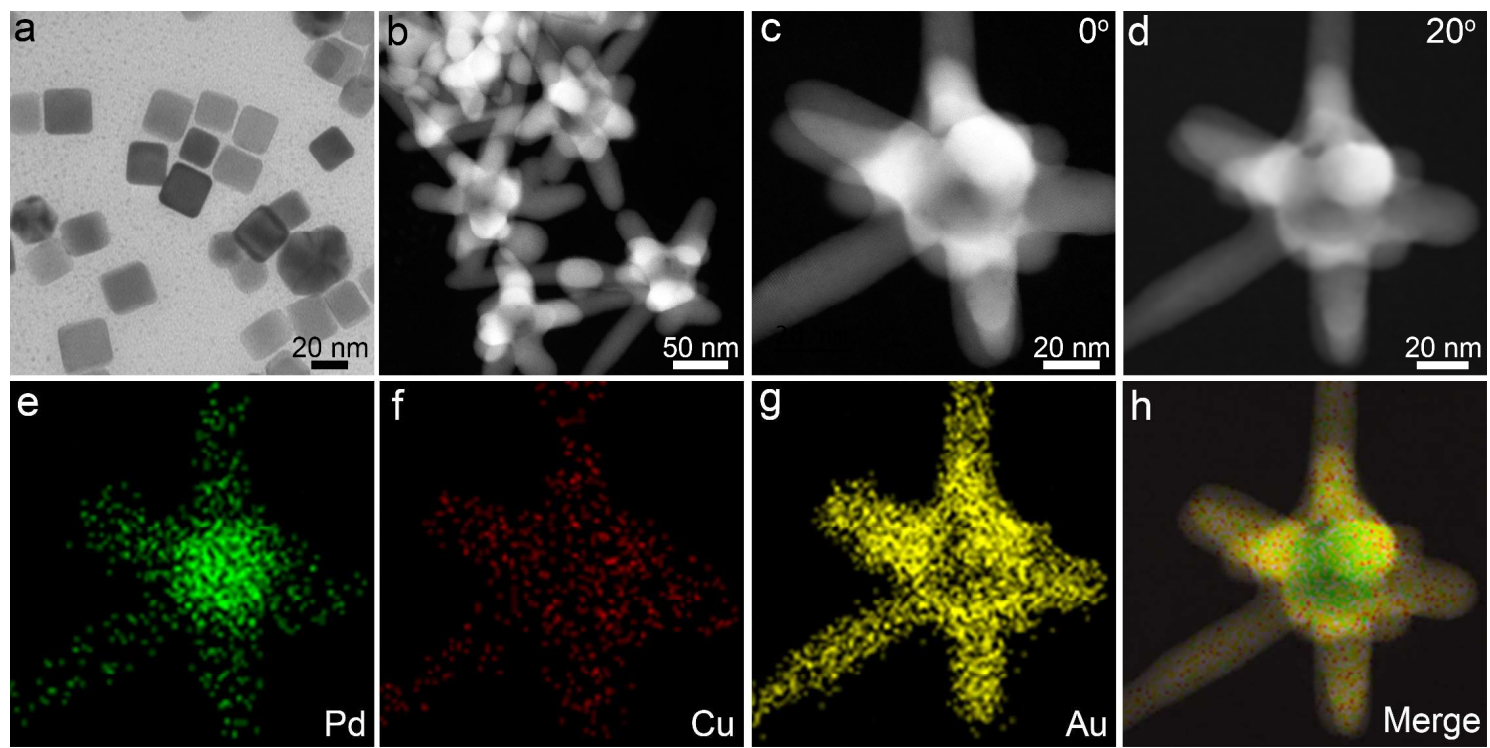

Figure S14. (a) TEM image of 22-nm Pd cubes with an aspect ratio of $\sim 1.0$. (b) HAADF-STEM image of three-dimensional Pd@AuCu multipods obtained using Pd cubic seeds shown in (a) with the other conditions unchanged. (c, d) HAADF-STEM images of an individual three-dimensional $\mathrm{Pd} @ \mathrm{AuCu}$ multipods recorded at different tilting angles: (c) $0^{\circ}$ and (d) $20^{\circ}$. (e-h) STEM-EDX elemental mapping of (e) $\mathrm{Pd}$, (f) $\mathrm{Cu}$, (g) $\mathrm{Au}$, and (h) merged images of the nanocrystal shown in $(\mathrm{d})$. 

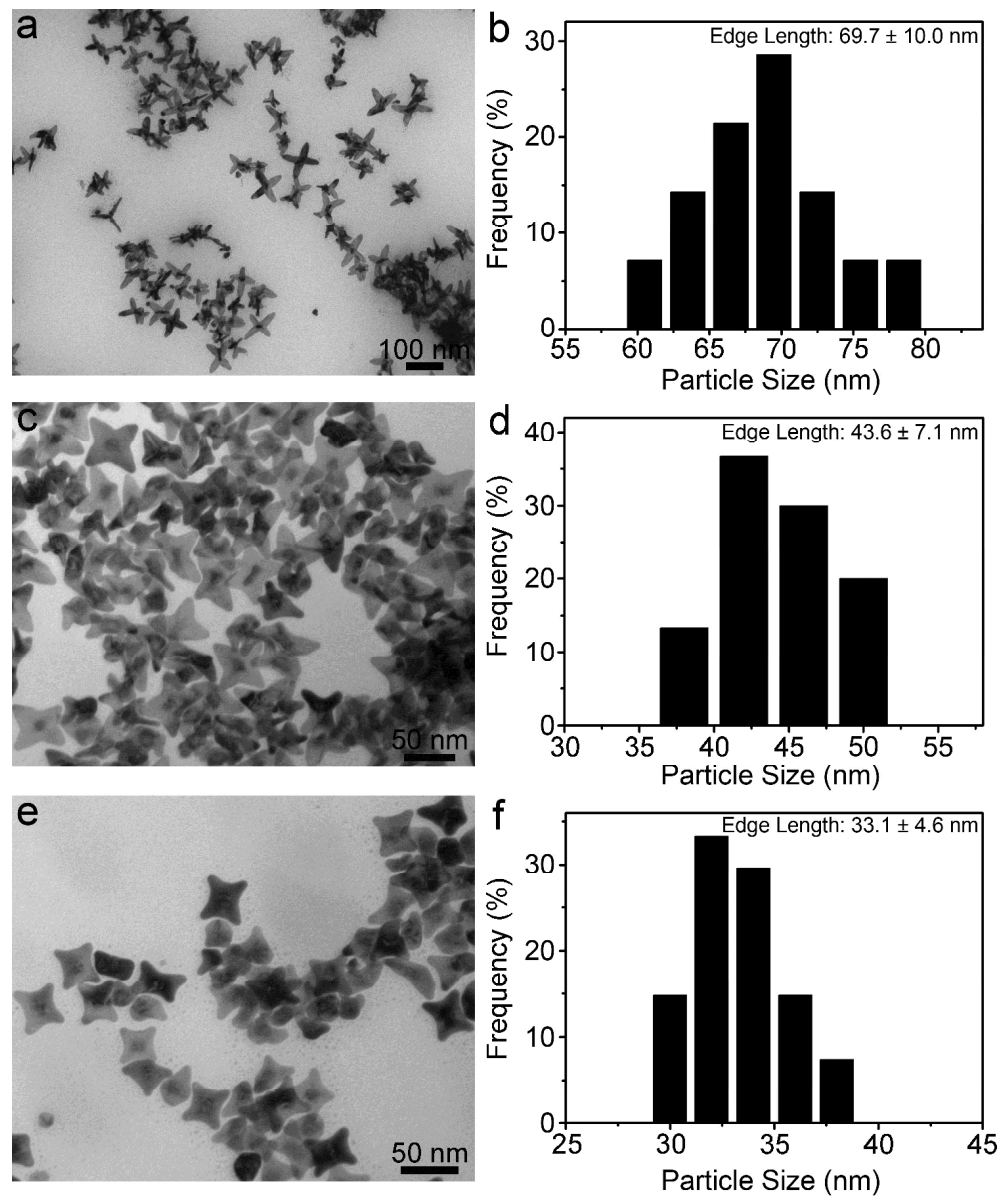

Figure S15. TEM images of the products obtained through the standard procedure except for using different volumes of 9-nm Pd cubic seeds: (a) $0.1 \mathrm{~mL}$, (c) $0.3 \mathrm{~mL}$, and (e) $0.5 \mathrm{~mL}$. (b, d, f) The corresponding histograms of size distribution. 

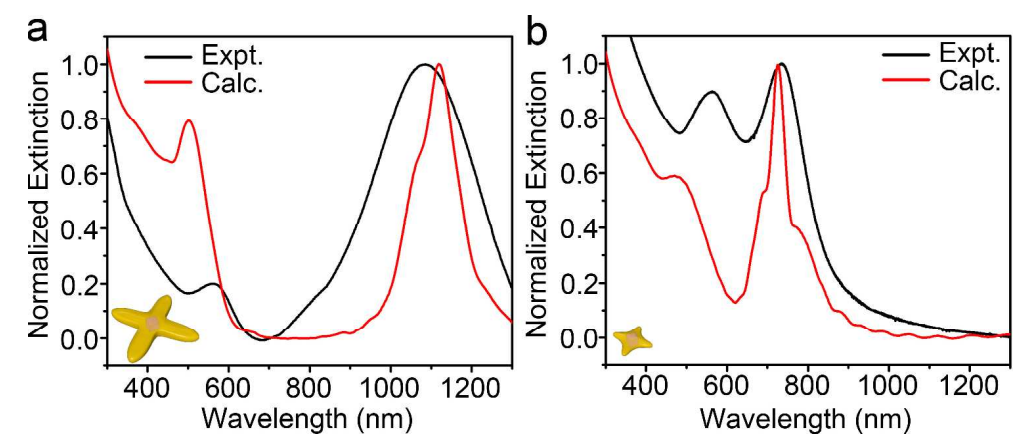

Figure S16. Comparison of experimental and calculated UV-Vis spectra of (a) 70- and (b) 33-nm Pd@AuCu core-shell planar tetrapods. 

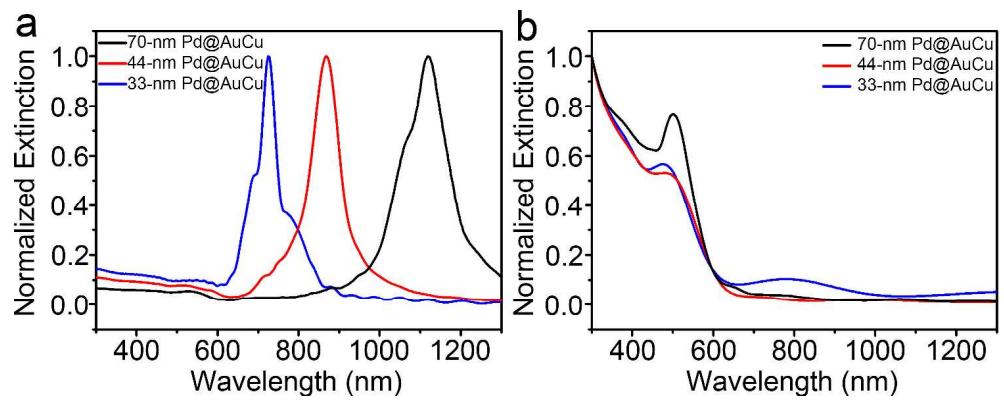

Figure S17. Calculated extinction spectra of $\mathrm{Pd} @ \mathrm{AuCu}$ core-shell planar tetrapods with different sizes excitated by polarized light along (a) $x$ - and (b) $z$-axis direction, respectively. 

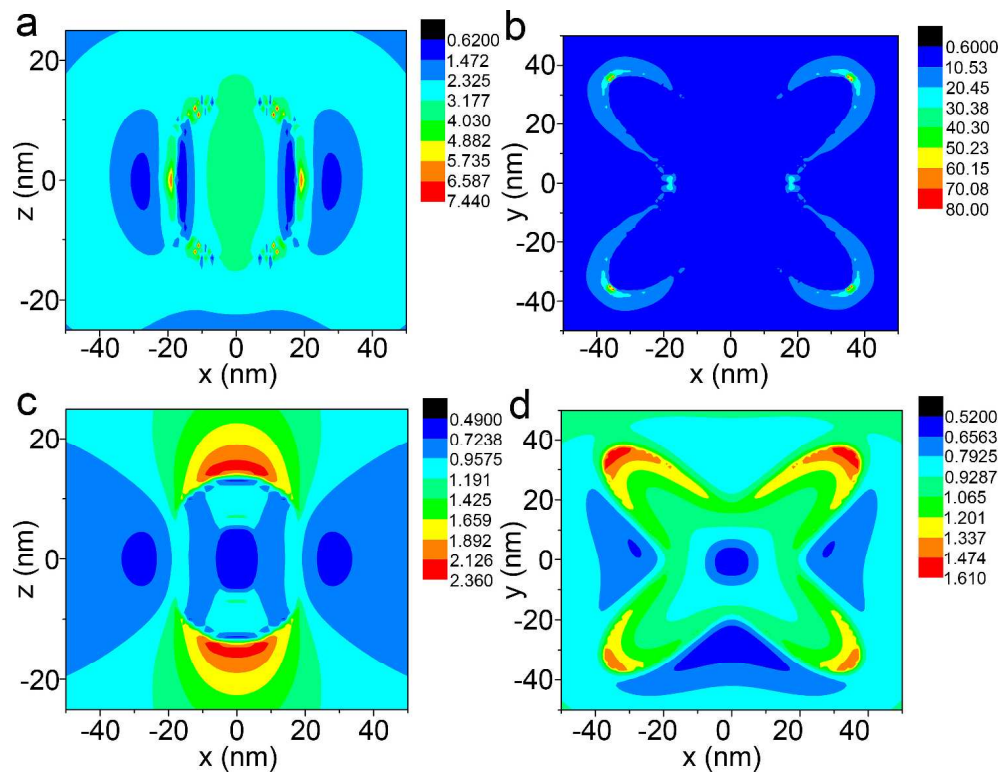

Figure S18. (a, b) Calculated near-field amplitude distributions at the interface of (a) $x z$ and (b) $x y$ for an individual 70-nm Pd@AuCu core-shell planar tetrapod at the 1100-nm resonance peak. (c, d) Calculated near-field amplitude distributions at the interface of (c) $x z$ and (d) $x y$ for an individual 70-nm Pd@AuCu core-shell planar tetrapod at the 500-nm resonance peak. 

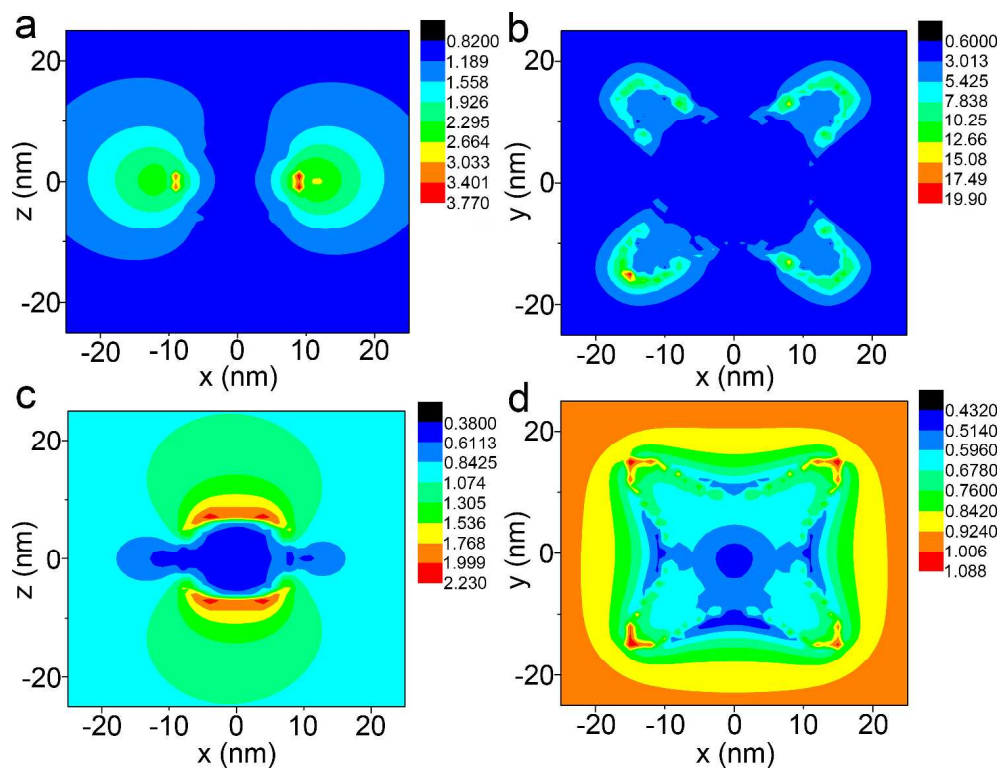

Figure S19. (a, b) Calculated near-field amplitude distributions at the interface of (a) $x z$ and (b) $x y$ for an individual 33-nm Pd@AuCu core-shell planar tetrapod at the 725-nm resonance peak. (c, d) Calculated near-field amplitude distributions at the interface of (c) $x z$ and (d) $x y$ for an individual 33-nm Pd@AuCu core-shell planar tetrapod at the 472-nm resonance peak. 


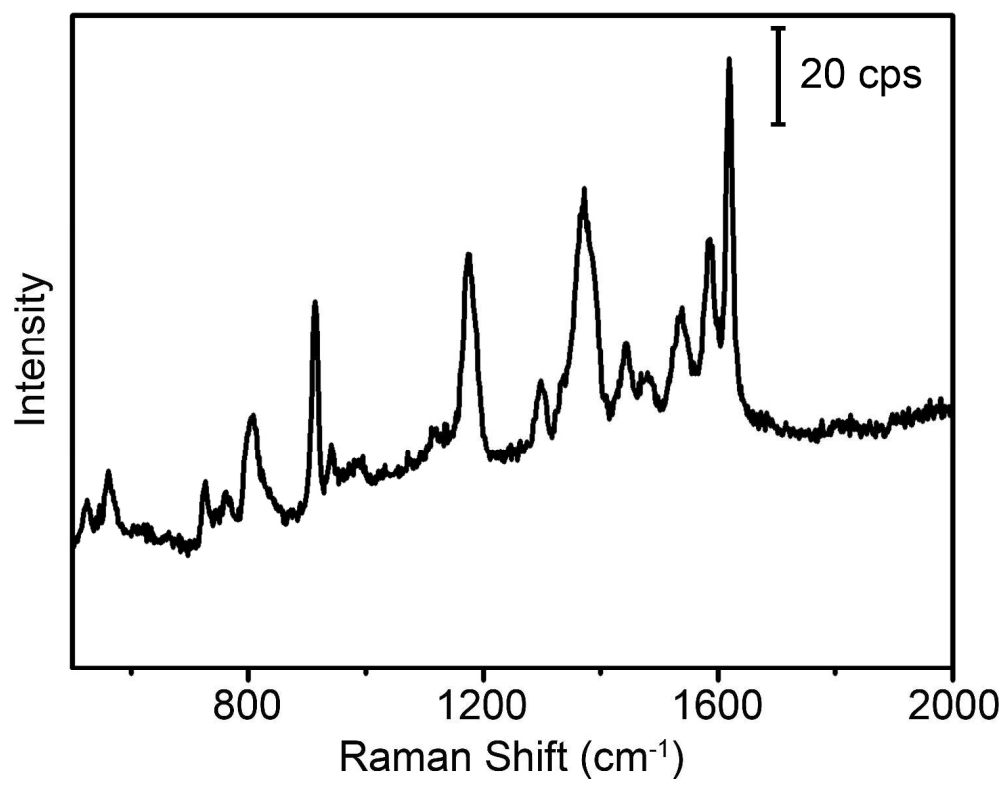

Figure S20. The normalized Raman spectrum of $10 \mu \mathrm{L}$ of a CV solution $\left(1.0 \times 10^{-3} \mathrm{M}\right)$ on the glass slide. The Raman spectrum was obtained with $\lambda_{\mathrm{ex}}=514 \mathrm{~nm}$ excitation, $\mathrm{P}_{\text {laser }}=1 \mathrm{~mW}$, and $\mathrm{t}$ $=30 \mathrm{~s}$. The scale bar is $20 \mathrm{cps}$.

\section{Estimation of the enhancement factor:}

The enhancement factor $(\mathrm{EF})$ of $\mathrm{Pd} @ \mathrm{AuCu}$ core-shell planar tetrapods on the substrate was calculated according to the following equation:

$$
\mathrm{EF}=\left(\mathrm{I}_{\mathrm{SERS}} / \mathrm{N}_{\mathrm{SERS}}\right) /\left(\mathrm{I}_{\mathrm{Nor}} / \mathrm{N}_{\mathrm{Nor}}\right)
$$

Where $\mathrm{I}_{\mathrm{SERS}}$ and $\mathrm{I}_{\text {Nor }}$ are the signal intensities of SERS and normal Raman spectra of CV at the same band (here, $\sim 1620 \mathrm{~cm}^{-1}$ ), and $\mathrm{N}_{\mathrm{SERS}}$ and $\mathrm{N}_{\mathrm{Nor}}$ represent the corresponding number of molecules in the focused incident laser spot. We assumed the CV molecules distributed evenly on the SERS substrates. As all the area of laser spot was the same, $\mathrm{N}_{\mathrm{SERS}}$ and $\mathrm{N}_{\mathrm{Nor}}$ can be substituted by the concentration of $\mathrm{CV}$, namely, $1.0 \times 10^{-6} \mathrm{M}$ and $1.0 \times 10^{-3} \mathrm{M}$. In Figure 4 and Figure S20, the $\mathrm{I}_{\mathrm{SERS}}$ and $\mathrm{I}_{\text {Nor }}$ were 640 cps for the 70-nm Pd@AuCu core-shell planar tetrapods and $71 \mathrm{cps}$ for $\mathrm{CV}$ at the Raman band of $\sim 1620 \mathrm{~cm}^{-1}$, respectively. Therefore, the EF was conservatively calculated to be $9.0 \times 10^{3}$ for 70-nm $\mathrm{Pd} @ \mathrm{AuCu}$ core-shell planar tetrapods. Similarly, the EFs were calculated to be $1.5 \times 10^{3}$ and $5.2 \times 10^{2}$ for 44-nm and 33-nm Pd@AuCu core-shell planar tetrapods, respectively. 